\title{
Wenshen decoction suppresses inflammation in IL-33-induced asthma murine model via inhibiting ILC2 activation
}

\author{
Weiling Huang ${ }^{1}$, Ying Song ${ }^{2}$, Lixin Wang ${ }^{3}$ \\ ${ }^{1}$ Department of Respiration, Shanghai Municipal Hospital of Traditional Chinese Medicine, Shanghai University of Traditional Chinese Medicine, \\ Shanghai 200071, China; ${ }^{2}$ Department of Pediatrics, Icahn School of Medicine at Mount Sinai, New York, NY, USA; ${ }^{3}$ Department of Integrated \\ TCM and Western Medicine, Shanghai Pulmonary Hospital, Tongji University, Shanghai 200433, China \\ Contributions: (I) Conception and design: W Huang, L Wang; (II) Administrative support: L Wang; (III) Provision of study materials or patients: \\ W Huang; (IV) Collection and assembly of data: W Huang, Y Song; (V) Data analysis and interpretation: W Huang; (VI) Manuscript writing: All \\ authors; (VII) Final approval of manuscript: All authors. \\ Correspondence to: Lixin Wang. Department of Integrated TCM and Western Medicine, Shanghai Pulmonary Hospital, Tongji University, 507 \\ Zhengmin Road, Shanghai 200433, China. Email: 1701005@tongii.edu.cn.
}

\begin{abstract}
Background: Wenshen decoction, consisting of Epimedium brevicornu Maxim, Morinda officinalis How and Cnidium monnieri (L.) Cusson, has favorable efficacy in the treatment of asthma in China.

Methods: The present study investigated the potential immunomodulatory mechanism underlying the therapeutic effects of Wenshen decoction on mouse asthma.

Results: Oral Wenshen decoction could ameliorate the production of IL-4, IL-5, IL-13 in the bronchoalveolar lavage fluid (BALF), reduce serum IgE, and improve the airway hyperresponsiveness (AHR) and airway inflammation in the $\mathrm{BALB} / \mathrm{c}$ mice after intranasal treatment with recombinant IL-33. Moreover, Wenshen decoction reduced ILC2 and ROR $\alpha$ mRNA expression, decreased the mRNA expression of ICOS and ST2 in the lung, but significantly increased the production of IFN- $\gamma$.

Conclusions: Our study indicates that Wenshen decoction may inhibit the activation of ILC2 through the IL-33/ST2/ICOS pathway to further suppress airway inflammation and AHR in the asthmatic mice, and the increased IFN- $\gamma$ might be related to the effects of Wenshen decoction on ILC2.
\end{abstract}

Keywords: Asthma; ILC2; traditional Chinese medicine; Wenshen decoction

Submitted May 22, 2019. Accepted for publication Aug 21, 2019.

doi: 10.21037/atm.2019.09.34

View this article at: http://dx.doi.org/10.21037/atm.2019.09.34

\section{Introduction}

Bronchial asthma is a chronic airway inflammatory disease characterized by airway hyperresponsiveness (AHR), and airflow obstruction involving multiple immune cells and inflammatory cells, such as T cell subsets, type 2 innate lymphoid cells, dendritic cells, and eosinophils $(1,2)$.

Classical acquired immune response dysfunction has long been recognized as the primary pathogenesis of asthma, and type 2 helper T lymphocytes (Th2) are the major actors in maintaining chronic airway inflammatory responses by secreting type 2 cytokines, such as interleukin-4 (IL-4), IL-5 and IL-13 (3). IL-5 can promote the activation and recruitment of airway eosinophils. IL-13 can induce goblet cell hyperplasia, mucus secretion and smooth muscle cell contraction, leading to AHR. IL-4 is able to facilitate the $\mathrm{T}$ cell differentiation and induce B-lymphocytes to release $\mathrm{IgE}$, causing immune response (4). However, these fail to explain many asthma-related phenomena.

In recent years, it has been found that innate immune responses and innate immune cells play important roles in the pathogenesis of asthma $(5,6)$. Group 2 innate lymphoid cells ILC2 are a subset of non-B cell, non-T cell family. It is independent of acquired immune system and can directly respond to a variety of external stimuli, secrete type 2 cytokines such as IL-4, IL-5 and IL-13, and trigger and maintain type 2 immune inflammatory response (7). ILC2 
not only has a potent ability to secrete Th2 type cytokines, but also participates in Th2-type immune responses (8), and can also interact with other immune cells (such as T cells) to mediate asthmatic inflammation (9-11).

ILC2 is also a retinoic acid receptor-related orphan receptor- $\alpha(\mathrm{ROR} \alpha)$ dependent ILC. ROR $\alpha$ is a key transcriptional regulator in the development of ILC2 and it not only mediates the immune response of ILC2 in the bone marrow and peripheral tissues, but also regulates the proliferation and biological function of ILC2 (12). It has been found that ILC 2 can be activated by a variety of stimuli, such as IL-33, IL-25 and thymic stromal lymphopoietin (TSLP) which are secreted by epithelial cells (13). Among them, IL-33 is a member of the IL- 1 family (14) and can act as a pro-inflammatory endogenous "danger signal" to activate various immune cells such as Th2 cells (15), ILC2 (16), dendritic cells (17), causing the increased expression of proinflammatory cytokines, chemokines, and lipid mediators, which therefore accelerates the progression of asthma (18). It has been revealed that IL-33 is highly expressed in the asthmatic patients, and its expression is positively related to asthma severity $(19,20)$. These suggest IL-33 bridges the innate immune system and the acquired immune system (21).

IL-33 exerts its function through the IL-33-bound IL-1 receptor like-1 component (IL-1R1; ST2). ST2 is expressed on a variety of cells such as ILC2, Th2 lymphocytes, and dendritic cells $(22,23)$. Besides ST2, ILC2 can express KLRGI, c-Kit, CD127, CD90 and CD45, but not express the markers of known immune lineages (24). ILC2 does not express RAG, and unlike T or B cells, ILC2 acts in a non-antigen-specific manner; however, ILC2 expresses inducible T cell costimulator (ICOS), which is an important costimulatory molecule in the T cells (25).

ICOS is a member of the CD28 family and highly expressed in the activated $\mathrm{T}$ cells and regulatory $\mathrm{T}$ cells, playing an important role in the survival and function of $\mathrm{T}$ cells, the differentiation of Th2 cells and the inflammatory response of the lung (26). Recent studies have shown that activated ILC2 can highly express ICOS, which substantially contributes to the production of IL-5, IL-13, bronchoalveolar lavage (BAL) eosinophilia and AHR. The binding of ICOS to ICOS-Ligand (ICOSL) not only plays an important role in the stabilization of function and maintenance of survival of ILC2, but also is necessary for the interaction between the ILC2 and other immune cells (27).

Wenshen decoction, which consists of Epimedium brevicornu Maxim, Morinda officinalis How and Cnidium monnieri (L.) Cusson, has been used for the treatment of asthma for a long time, achieving favorable efficacy (such as reduced frequencies of asthma recurrence and colds) in China. Although few studies have been conducted to investigate the effects of Wenshen decoction on the asthma, the application of related monomers in asthma has been confirmed both in animal models and cell experiments. In vivo, it has been found that icariin can improve asthma by regulating the immune imbalance of Th17/Treg (28), as well as reducing the release of inflammatory factors via up-regulating the glucocorticoid receptor (29). Osthole is one of the main components of Cnidium, and possess anti-inflammatory, anti-allergic and anti-hypertensive activities. Studies have shown that, in OVA-induced asthma mouse model, osthole can inhibit the activation of nuclear factor- $\kappa \mathrm{B}(\mathrm{NF}-\kappa \mathrm{B})$ signaling pathway, and reduce the Th2 cytokines and $\operatorname{IgE}$ in the serum and $\operatorname{BALF}(30,31)$.

To date, studies have confirmed that Chinese medicines have a unique immunoregulatory role in the asthma, but little is known about the regulation of innate immune cells by traditional Chinese medicines (32-34). ILC2 is a new subset of innate immune cells identified in recent years, and plays an important role in the airway inflammation in case of asthma. However, most studies focus on the mechanism of ILC2, and little is known about the influence of clinical drugs, especially the traditional Chinese medicine, on the ILC2. IL-33 is a factor closely related to the activation of ILC2. Our previous study revealed that Wenshen decoction could down-regulate the expression of IL-33 and ST2 and reduce the release of Th2 cytokines in OVA-induced asthmatic mice. The present study was to investigate whether Wenshen decoction could suppress airway inflammation via ILC2 to ameliorate asthma in the IL-33-induced asthma mouse model. Our findings may provide new evidence on the clinical use for traditional Chinese medicine-Wenshen decoction in the adjunctive treatment of asthma.

\section{Methods}

\section{Mice}

Thirty female BALB/c mice aged 6- 8 weeks were purchased from the Shanghai Slack Laboratory Animal Center and maintained in the specific pathogen-free environment of the Animal Center of Shanghai Pulmonary Hospital. All animals were allowed to acclimatize for 7 days prior to experiment, maintained at $20-23{ }^{\circ} \mathrm{C}$ with a $12 \mathrm{~h} / 12 \mathrm{~h}$ light-dark cycle and given ad libitum access to water and 


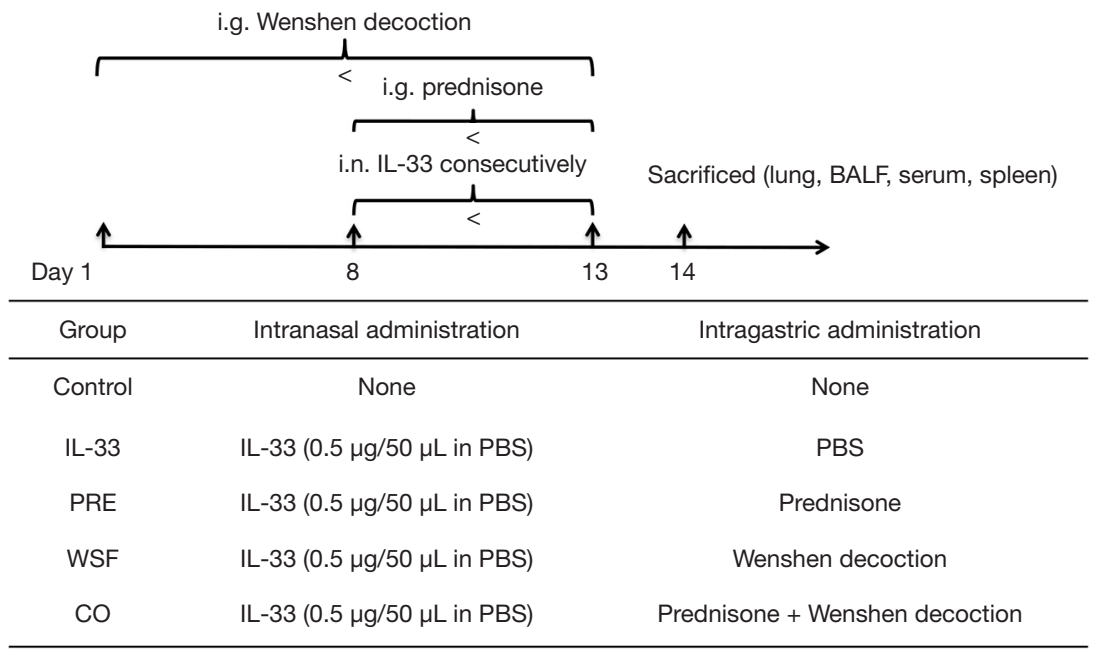

Figure 1 Experimental protocol for IL-33-induced asthmatic model and treatments.

food. The animal care and protocol were approved by the Animal Experiment Administration Committee of Shanghai Municipal Hospital of TCM (No. 2019DWLL002). These animals were randomly divided into 5 groups $(\mathrm{n}=6$ per group): control group, IL-33 group (IL-33 group), IL$33+$ prednisone group (PRE group), IL-33 + Wenshen decoction group (WSF group), and IL-33 + prednisone + Wenshen decoction group (CO group).

\section{Preparation of Wenshen decoction and prednisone}

Wenshen decoction was combined with Epimedium brevicornu Maxim (Herba Epimedii), Cnidium monnieri (L.) Cuss and Morinda officinalis How. These herb pieces were purchased from Shanghai Municipal Hospital of Traditional Chinese Medicine, mixed at the ratio of 1:1:1 $(\mathrm{w} / \mathrm{w})$, soaked in 8 volumes of distilled water for $30 \mathrm{~min}$, and decocted for $1 \mathrm{~h}$ twice. The decoction was then filtrated through a sieve, and the filtrate was collected and stored at $-80{ }^{\circ} \mathrm{C}$. Prednisone was purchased from Shanghai Sine Pharmaceutical Laboratories Co., Ltd.

\section{Asthmatic mouse model induced by IL-33 and drug treatment}

The asthmatic model was established by intranasal administration (i.n.) of recombinant murine IL-33 (0.5 $\mu \mathrm{g}$ per mouse, BioLegend) for 6 consecutive days. To examine the effect of Wenshen decoction and prednisone on the asthma, mice in the WSF group were intragastrically treated with WSF at $13.5 \mathrm{~g} / \mathrm{kg}$ body weight since 7 days before the first i.n IL-33 treatment, and the prednisone was intragastrically administered at $1.5 \mathrm{mg} / \mathrm{kg}$ body weight since the first day of i.n IL-33 treatment. In the control group, mice were not treated with IL-33. The grouping and treatments are shown in the Figure 1.

\section{Measurement of $A H R$}

Mice in each group were tested for airway reactivity within $24 \mathrm{~h}$ after the last intranasal administration. In brief, the mice were anesthetized with $2 \%$ sodium pentobarbital, then allowed to inhale methacholine at different concentrations $(0,3.125,6.25$ and $12.5 \mathrm{mg} / \mathrm{mL})$ (Mch, Sigma-Aldrich, USA) successively followed by the detection of airway responsiveness for $3 \mathrm{~min}$. The invasive pulmonary function detection was performed with a small animal lung function detection system (FinePointe RC System, Buxco, USA). The airway responses are expressed as the change in airway resistance.

\section{Collection of bronchoalveolar lavage fluid (BALF)}

The mice were sacrificed by cervical dislocation after detection of airway responsiveness. The right main bronchus was ligated, the trachea was opened, and then a catheter was inserted into the left main bronchus. Into the left lung, $0.3 \mathrm{~mL}$ of saline was injected through a catheter for lavage. This procedure was repeated twice. $70-80 \%$ of saline was retrieved. The BALF was then centrifuged at 3,000 r/min 
Table 1 Primer sequences used for qPCR amplification

\begin{tabular}{|c|c|c|}
\hline Gene & Primer & Synthesized sequence \\
\hline \multirow[t]{2}{*}{$\beta$-actin } & $\begin{array}{l}\text { Forward } \\
\text { primer }\end{array}$ & 5'-AGTGTGACGTTGACATCCGT-3' \\
\hline & $\begin{array}{l}\text { Reverse } \\
\text { primer }\end{array}$ & 5'-GCAGCTCAGTAACAGTCCGC-3' \\
\hline \multirow[t]{2}{*}{$R O R \alpha$} & $\begin{array}{l}\text { Forward } \\
\text { primer }\end{array}$ & 5'-GATTGAAAACTACCAGAACAAGCAG-3' \\
\hline & $\begin{array}{l}\text { Reverse } \\
\text { primer }\end{array}$ & 5'-GCACATCCTAATAAACACCACCTC-3' \\
\hline \multirow[t]{2}{*}{ ST2 } & $\begin{array}{l}\text { Forward } \\
\text { primer }\end{array}$ & 5'-CTGGCACTGGCATTTGGT-3' \\
\hline & $\begin{array}{l}\text { Reverse } \\
\text { primer }\end{array}$ & 5'-GCCTACGAGCAGGAGATT-3' \\
\hline \multirow[t]{2}{*}{ ICOS } & $\begin{array}{l}\text { Forward } \\
\text { primer }\end{array}$ & 5'-TTCCCTTGGTCTTGGTGAGTT-3' \\
\hline & $\begin{array}{l}\text { Reverse } \\
\text { primer }\end{array}$ & 5'-GTGACTTACTACCTGCCTTAC-3' \\
\hline
\end{tabular}

for $10 \mathrm{~min}$ at $4{ }^{\circ} \mathrm{C}$, and the supernatant was collected and stored at $-80^{\circ} \mathrm{C}$. The remaining cells were re-suspended with $100 \mu \mathrm{L}$ of PBS for cell counting on an automatic hematology analyzer (BC-5300 Vet, Mindray, China).

\section{Lung histopathology}

The right lung was collected and fixed in $4 \%$ paraformaldehyde solution after collection of BALF. Then, the lung tissues were dehydrated, embedded in paraffin, cut into 4- $\mu \mathrm{m}$ sections, and stained with hematoxylin-eosin (HE). The pathology of lung tissues was examined under a light microscope.

\section{Detection of cytokine and IgE levels by ELISA}

The supernatant of BALF and serum were processed for the measurement of cytokines, including IL-4, IL-5, IL-13 and IgE by ELISA (BioLegend, USA) according to the manufacturer's instructions.

\section{Real-time quantitative PCR}

Total RNA was extracted from the lungs using Trizol reagent (Invitrogen, USA) and complementary DNA was synthesized using the PrimeScript ${ }^{\mathrm{TM}}$ RT Master Mix (Takara Japan) according to the manufacturer's instructions. RT-qPCR was performed with the SYBR Premix Ex Taq ${ }^{\mathrm{TM}}$ II (Takara,
Japan) on the QuantStudio 6 flex Real-Time PCR System (Life Technologies, USA). The primers used for qPCR were synthesized in Shanghai Sangon Biotech Co., Ltd (Shanghai, China) and are shown in Table 1. The expression of genes was determined using the $2^{-\Delta \Delta \mathrm{Ct}}$ method and normalized to the expression of $\beta$-actin in the same sample.

\section{Flow cytometric assay of ILC2, Tb1cells and DC}

The spleen and lung were collected from each mouse and placed in RPMI1640 medium (Sigma-Aldrich, USA). A single cell suspension was prepared. For ILC2 assay, cells were incubated with PerCP-Cy ${ }^{\mathrm{TM}} 5.5-$ Mouse Lineage Antibody Cocktail (BD Pharmingen ${ }^{\mathrm{TM}}$, USA), APC-antimouse KLRG1 (BioLegend, USA), and PE-anti-mouse ST2 (BioLegend, USA). For Th1 cells assay, cells were incubated with PerCP-Cy ${ }^{\mathrm{TM}} 5.5$-anti-mouse CD4 (BD Pharmingen ${ }^{\mathrm{TM}}$, USA), and FITC-anti-mouse IL-17A (BD Pharmingen ${ }^{\mathrm{TM}}$, USA). After washing twice, cells were subjected to flowcytometry on the FACScan (BD Biosciences, USA). The data were analyzed using Flowjo software version 7.6.1.

\section{Statistical analysis}

All the data are presented as mean \pm standard deviation $(\mathrm{SD})$. Data among at least three independent experiments were compared using one-way analysis of variance (ANOVA). A value of $\mathrm{P}<0.05$ was considered statistically significant.

\section{Results}

\section{Effect of Wenshen decoction on airway resistance in asthmatic mice}

One of the main manifestations of asthma is AHR. In order to study the effect of Wenshen decoction on the AHR in asthmatic mice, airway resistance was detected within $24 \mathrm{~h}$ after the last intranasal administration of IL-33. Results showed there was no significant difference in the airway resistance between the groups when Mch at $3.125 \mathrm{mg} / \mathrm{mL}$ was inhaled. The airway resistance in the IL-33 model group was significantly higher than in the control group when Mch at $6.25 \mathrm{mg} / \mathrm{mL}$ was inhaled $(\mathrm{P}<0.01)$, and different treatments tended to down-regulate the airway resistance as compared to the IL-33 model group although there was no significant difference $(\mathrm{P}>0.05)$. In case of inhalation of Mch at $12.5 \mathrm{mg} / \mathrm{mL}$, the airway resistance in the IL-33 model group increased markedly as compared to 


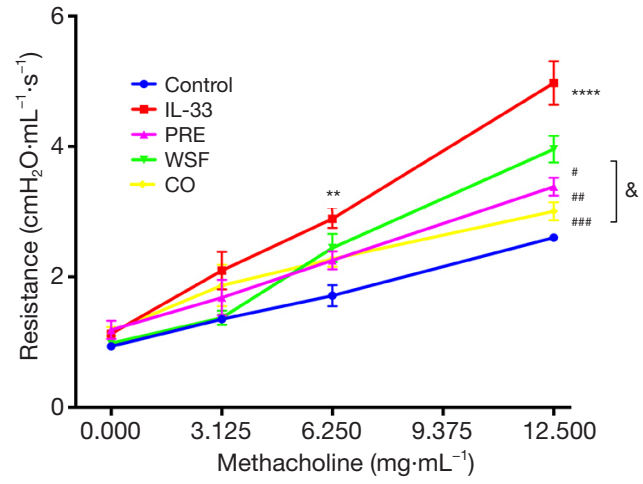

Figure 2 Effect of Wenshen decoction on the airway resistance in mice under different concentrations of methacholine. Data are show as means $\pm \operatorname{SEM}(\mathrm{n}=6) .{ }^{* *} \mathrm{P}<0.01$, ${ }^{* * *} \mathrm{P}<0.0001$, control group vs. IL-33 group; ${ }^{\#} \mathrm{P}<0.05,{ }^{\#} \mathrm{P}<0.01,{ }^{\# \#} \mathrm{P}<0.001$, PRE, WSF, and $\mathrm{CO}$ groups vs. IL-33 group; ${ }^{8} \mathrm{P}<0.05, \mathrm{CO}$ group vs. WSF group. Control, control group; IL-33, IL-33-treated group; PRE, prednisone acetate group; WSF, Wenshen decoction group; CO, prednisone and Wenshen decoction group.

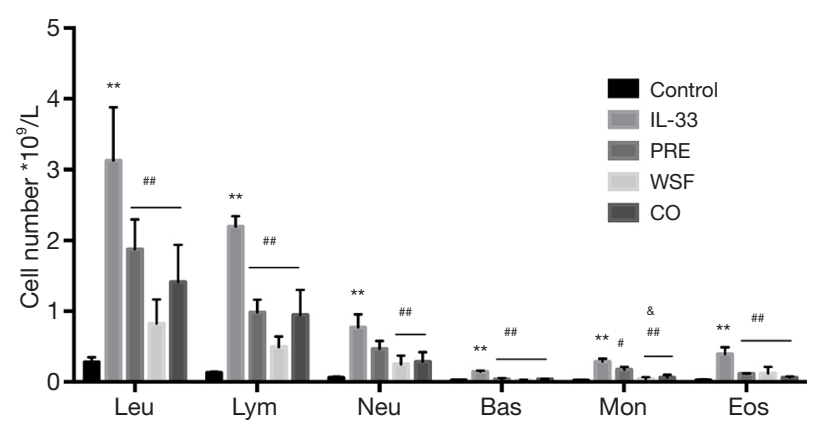

Figure 3 Effect of Wenshen decoction on the BALF inflammatory cells. At $24 \mathrm{~h}$ after the last IL-33-challenge, the total cell counts and differential cell counts were determined. Data are show as means $\pm \operatorname{SEM}(\mathrm{n}=6) .{ }^{*} \mathrm{P}<0.01$, control group vs. IL-33 group; ${ }^{\#} \mathrm{P}<0.05,{ }^{\#} \mathrm{P}<0.01, \mathrm{PRE}, \mathrm{WSF}$, and CO groups vs. IL-33 group; ${ }^{\circledR} \mathrm{P}<0.05, \mathrm{PRE}$ group vs. WSF group, $\mathrm{PRE}$ group vs. CO group. Control, control group; IL-33, IL-33-treated group; PRE, prednisone acetate group; WSF, Wenshen decoction group; CO, prednisone and Wenshen decoction group; Leu, white blood cells; Lym, lymphocytes; Neu, neutrophils; Bas, basophils; Mon, monocytes; Eos, eosinophils. BALF, bronchoalveolar lavage fluid.

the control group $(\mathrm{P}<0.001)$, and different treatments also significantly down-regulated the airway resistance $(\mathrm{P}<0.05)$. In addition, the airway resistance significantly reduced in the $\mathrm{CO}$ group as compared to the WSF group $(\mathrm{P}<0.05)$; this indicates that $\mathrm{CO}$ treatment is better to attenuate the airway resistance in the asthmatic mouse model (Figure 2).

\section{Effect of Wenshen decoction on eosinophilia and BAL fluid composition}

At $24 \mathrm{~h}$ after the last intranasal administration of IL-33, BALF was collected for the detection of eosinophilia and BAL fluid composition. As shown in Figure 3, the numbers of leukocytes, eosinophils, basophils, neutrophils, lymphocytes, and monocytes in the IL-33 group increased significantly compared with the control group. $(\mathrm{P}<0.01)$; after different treatments, the numbers of these cells significantly reduced as compared to the IL-33 group $(\mathrm{P}<0.01)$, except for the neutrophils in the prednisone group $(\mathrm{P}>0.05)$; there were no significant differences in the numbers of lymphocytes, leukocytes, eosinophils, basophils, neutrophils among the WSF group, the PRE group and the $\mathrm{CO}$ group $(\mathrm{P}>0.05)$, but the number of monocytes decreased significantly in the WSF group and the CO group when compared with the PRE group $(\mathrm{P}<0.05)$.

\section{Effect of Wenshen decoction on pathology of lung tissue in mice}

At $24 \mathrm{~h}$ after the last intranasal administration of IL-33, the right upper lobe of the lung was fixed in 4\% paraformaldehyde for $48 \mathrm{~h}$, followed by HE staining. As shown in Figure 4, in the control group, the tracheal lumen was smooth, there were no obvious thickening of the tracheal wall and no inflammatory cell infiltration around the wall and the epithelial cells were relatively complete in the lung. Compared with the control group, the tracheal wall in the IL-33 group was significantly thickened, and there was a large amount of inflammatory cells around the lumen, mainly eosinophils, and the goblet cells proliferated in the bronchial epithelium. Compared with the IL-33 group, the bronchial wall was relatively thinner and the inflammatory cells infiltrated around the wall significantly reduced in the PRE group, WSF group and CO group.

\section{Effect of Wenshen decoction on cytokines in mouse BALF and IgE in serum}

Inflammatory cytokines play an important role in the pathogenesis of asthma. This study examined the effect of Wenshen decoction on the expression of IL-4, IL-5, IL-13 in the BALF and IgE in the serum. As shown in Figure 5, the BALF contents of IL-4, IL-5 and IL-13 in the IL-33 


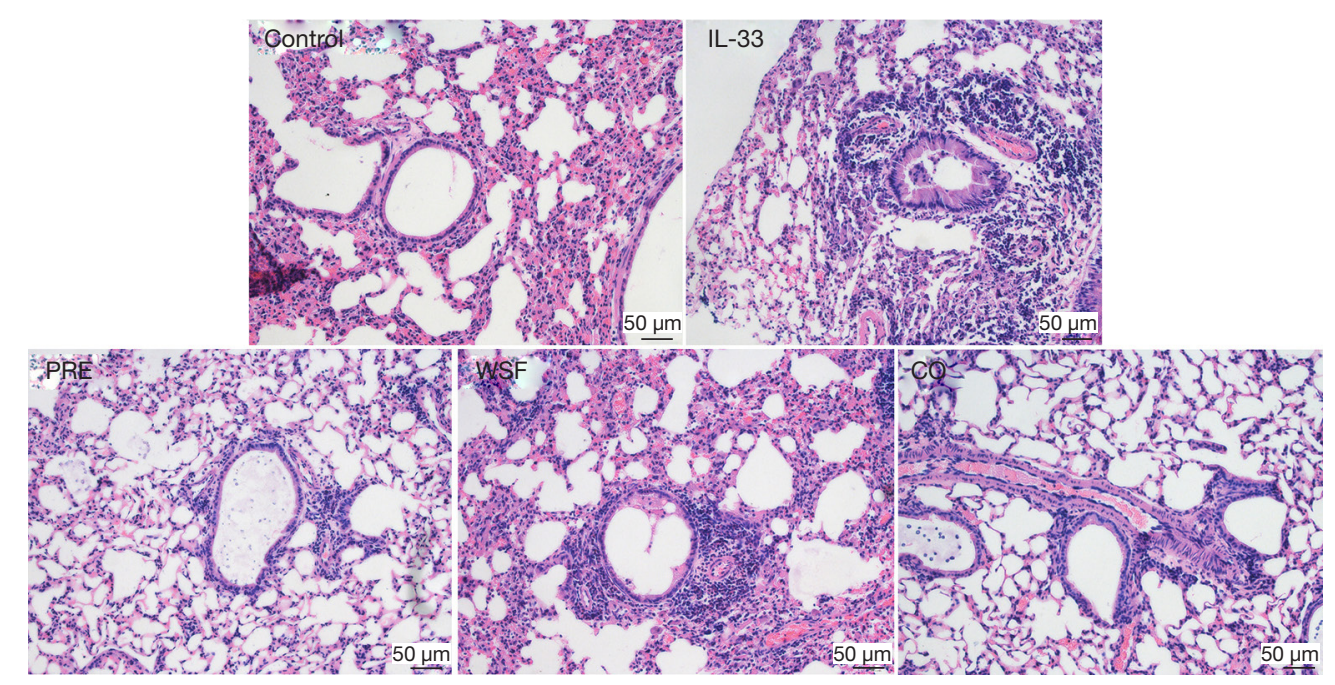

Figure 4 Effect of Wenshen decoction on the histopathology of the lung. Inflammation was evaluated in the lung by analyzing inflammatory cell infiltration after H\&E staining. Magnification: 200x. Control, control group; IL-33, IL-33-treated group; PRE, prednisone acetate group; WSF, Wenshen decoction group; CO, prednisone and Wenshen decoction group.


Figure 5 Effect of Wenshen decoction on the BALF IL-4 (B), IL-5 (C) and IL-13 (D) in BALF and serum IgE (A). At 24 h after the last IL-33 challenge, BALF and serum were collected for the measurement of these cytokines by ELISA. Data are show as means \pm SEM ( $=6)$. ${ }^{* *} \mathrm{P}<0.01,{ }^{* * *} \mathrm{P}<0.001$, control group vs. IL-33 group; ${ }^{\# \#} \mathrm{P}<0.01, \mathrm{PRE}, \mathrm{WSF}$, and $\mathrm{CO}$ groups vs. IL-33 group; ${ }^{\text {* }} \mathrm{P}<0.05,{ }^{\& \&} \mathrm{P}<0.01, \mathrm{PRE}$ group vs. WSF group, PRE group vs. CO group. Control, control group; IL-33, IL-33-treated group; PRE, prednisone acetate group; WSF, Wenshen decoction group; CO, prednisone and Wenshen decoction group. BALF, bronchoalveolar lavage fluid. 

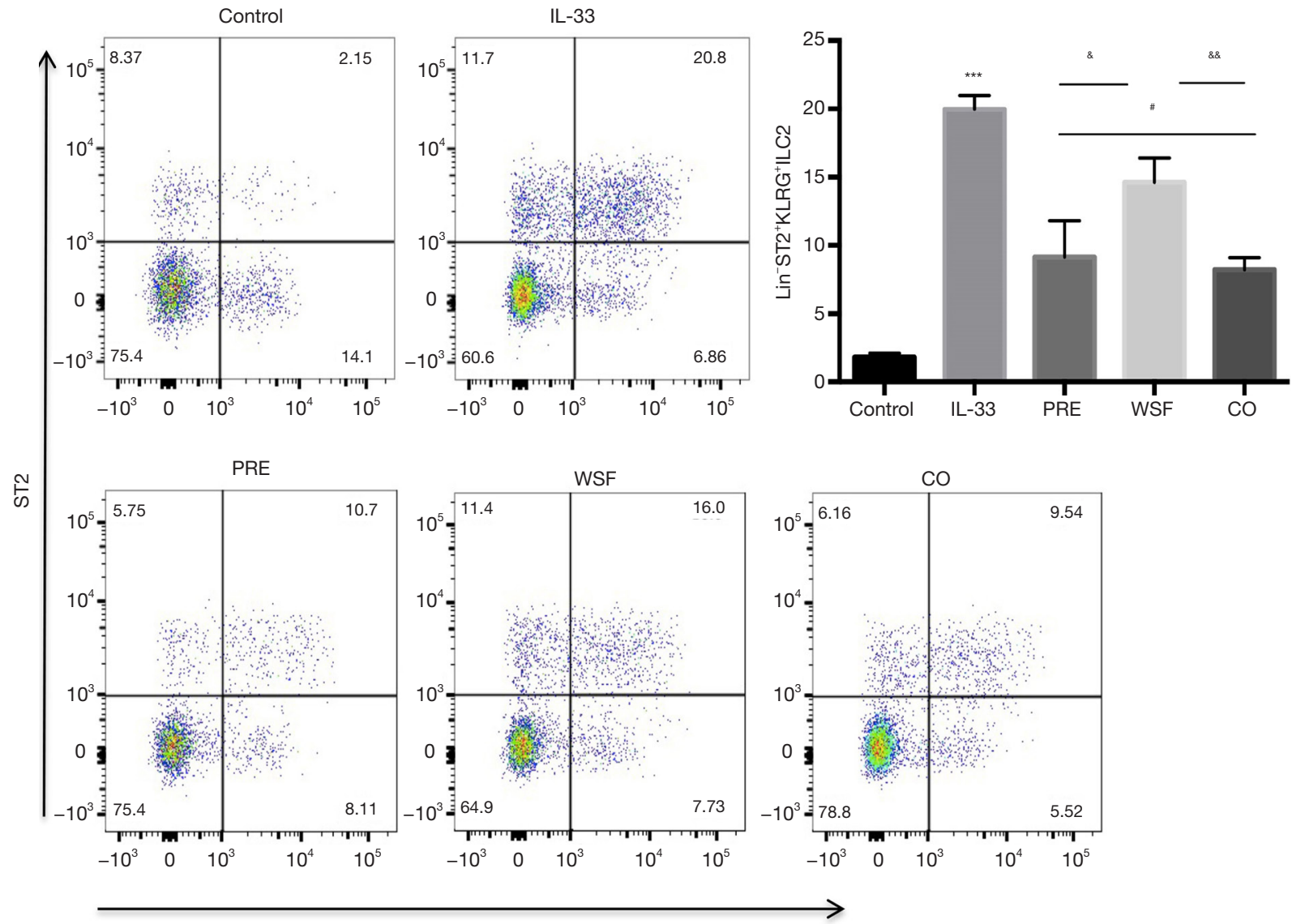

KLRG1

Figure 6 Effect of Wenshen decoction on ILC2 in the lung. Data are show as means $\pm \mathrm{SEM}(\mathrm{n}=6)$. ${ }^{* *} \mathrm{P}<0.001$, control group $v s$. IL-33 group; ${ }^{\mathrm{P}}<0.05$, PRE, WSF, and CO groups vs. IL-33 group; ${ }^{\circledR} \mathrm{P}<0.05,{ }^{8}{ }^{\star} \mathrm{P}<0.01$, WSF group vs. CO group. Control, control group; IL-33, IL-33-treated group; PRE, prednisone acetate group; WSF, Wenshen decoction group; CO, prednisone and Wenshen decoction group.

group were significantly higher than in the control group $(\mathrm{P}<0.01)$; compared with IL-33 group, different treatments could significantly reduce the contents of IL-4, IL-5 and IL-13 $(\mathrm{P}<0.01)$. The down-regulated production of IL-5 in the PRE group was more obvious than in the WSF group $(\mathrm{P}<0.01)$, and the IL-13 content was lower in the CO group than in the WSF group $(\mathrm{P}<0.05)$. This indicates Wenshen Decoction can down-regulate the contents of inflammatory cytokines (IL-4, IL-5 and IL-13) in the BALF. Compared with the control group, the serum IgE content significantly increased in the IL-33 group $(\mathrm{P}<0.05)$; however, the serum $\mathrm{IgE}$ content reduced significantly in the PRE group, WSF group and $\mathrm{CO}$ group $(\mathrm{P}<0.05)$.

\section{Effect of Wenshen decoction on ILC2 in lung tissue of mice}

At $24 \mathrm{~h}$ after the last intranasal administration of IL-33, the left lung was processed for flow cytometer. As shown in Figure 6, IL-33 significantly increased the LIN-ST2 + KLRG1 + ILC2 cells compared with the control group $(\mathrm{P}<0.01)$; compared with the IL-33 group, the LIN-ST2 + KLRG1 + ILC2 cells significantly reduced after different treatments $(\mathrm{P}<0.05)$, but the reduction of LIN-ST2 + KLRG1 + ILC2 cells in the WSF group was the lowest.

\section{Effects of Wenshen decoction on $m R N A$ expression of RORa, ICOS ST2, GATA-3 and T-bet in the lung}

At $24 \mathrm{~h}$ after the last intranasal administration of IL-33, RNA was extracted from the right lung for the detection of mRNA expression of ROR $\alpha$, ICOS ST2, GATA-3 and T-bet. Results showed, compared with the control group, the mRNA expression of ICOS, ST2, GATA-3 and ROR $\alpha$ in the lung of IL-33 group increased markedly $(\mathrm{P}<0.01)$; 
A

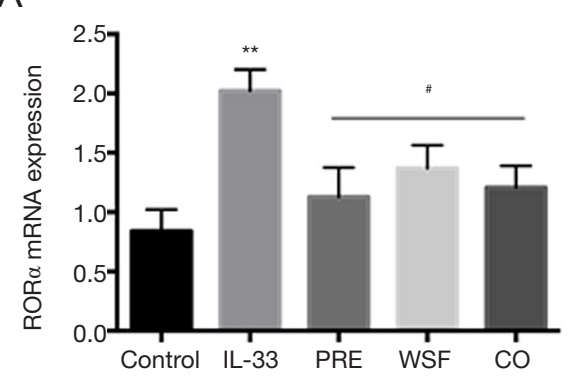

\section{$\mathrm{D}$}

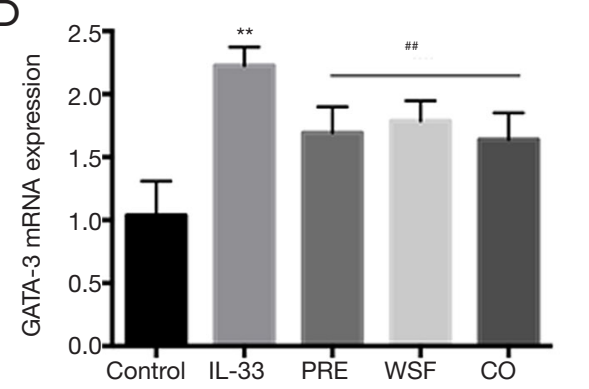

B

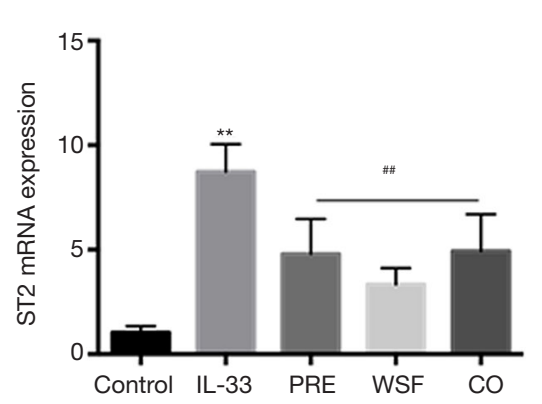

E

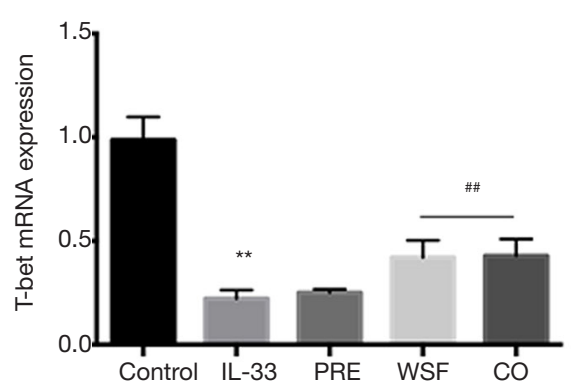

C

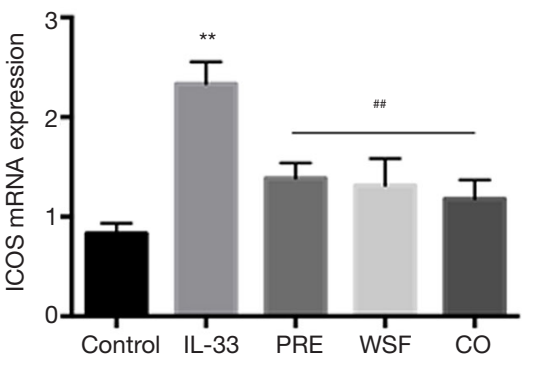

Figure 7 Effect of Wenshen decoction on mRNA expression of ROR $\alpha$ (A), ST2 (B), ICOS (C), GATA-3 (D) and T-bet (E). Data are show as means $\pm \mathrm{SEM}(\mathrm{n}=6)$. ${ }^{* *} \mathrm{P}<0.01$, control group vs. IL-33 group; ${ }^{\#} \mathrm{P}<0.05,{ }^{\# \#} \mathrm{P}<0.01, \mathrm{PRE}$, WSF, and CO groups vs. IL-33 group. Control, control group; IL-33, IL-33-treated group; PRE, prednisone acetate group; WSF, Wenshen decoction group; CO, prednisone and Wenshen decoction group. ROR $\alpha$, receptor-related orphan receptor- $\alpha$.

compared with the IL-33 group, the mRNA expression of ICOS, ST2, GATA-3 and ROR $\alpha$ was significantly downregulated in the PRE group, WSF group and CO group $(\mathrm{P}<0.05)$. On the contrary, the mRNA expression of T-bet in the lung of IL-33 group decreased markedly $(\mathrm{P}<0.01)$; compared with the IL-33 group, the mRNA expression of T-bet was significantly up-regulated in the WSF group and CO group $(\mathrm{P}<0.05)$, however, there was no effect of PRE group on T-bet (Figure 7).

\section{Effects of Wenshen decoction on IFN-y expression}

At $24 \mathrm{~h}$ after the last treatment with IL-33, the expression of Th1 in the spleen and the antigen presentation function of DCs in the lungs were examined by flow cytometry. As shown in Figure 8, compared with the control group, IL-33 had no significant effect on the expression of IFN- $\gamma$ ( $>0.05)$; compared with IL-33 group, prednisone failed to significantly down-regulate IFN- $\gamma$ expression. In contrast, the Wenshen decoction and the combination treatment significantly up-regulated the expression of IFN- $\gamma$ $(\mathrm{P}<0.05)$.

\section{Discussion}

Bronchial asthma is a recurrent chronic inflammatory disease with increasing morbidity and mortality (2), which significantly threatens the life and health of humans. Currently, corticosteroids are the first line medicine for the treatment of asthma according to the Global Initiative for Asthma (GINA) Program, because they are the most effective to prevent asthma attacks (35). However, longterm use of corticosteroids has many side effects, such as full moon face, buffalo back, osteoporosis, and potential drug resistance, causing intractable asthma. Therefore, it is imperative to develop new medicines to control asthma. Cheerfully, the Traditional Chinese Medicine has been accepted as an alternative therapy for asthma because of its favorable efficacy and safety for asthma.

Wenshen decoction consists of Epimedium brevicornu Maxim, Morinda officinalis How and Cnidium monnieri (L.) Cusson, has achieved efficacy in the treatment of asthma in China, although few studies have been conducted to its effect and mechanism in asthmatic animals. The present study investigated the immunomodulatory effects of Wenshen decoction in the IL-33-induced mouse asthma 

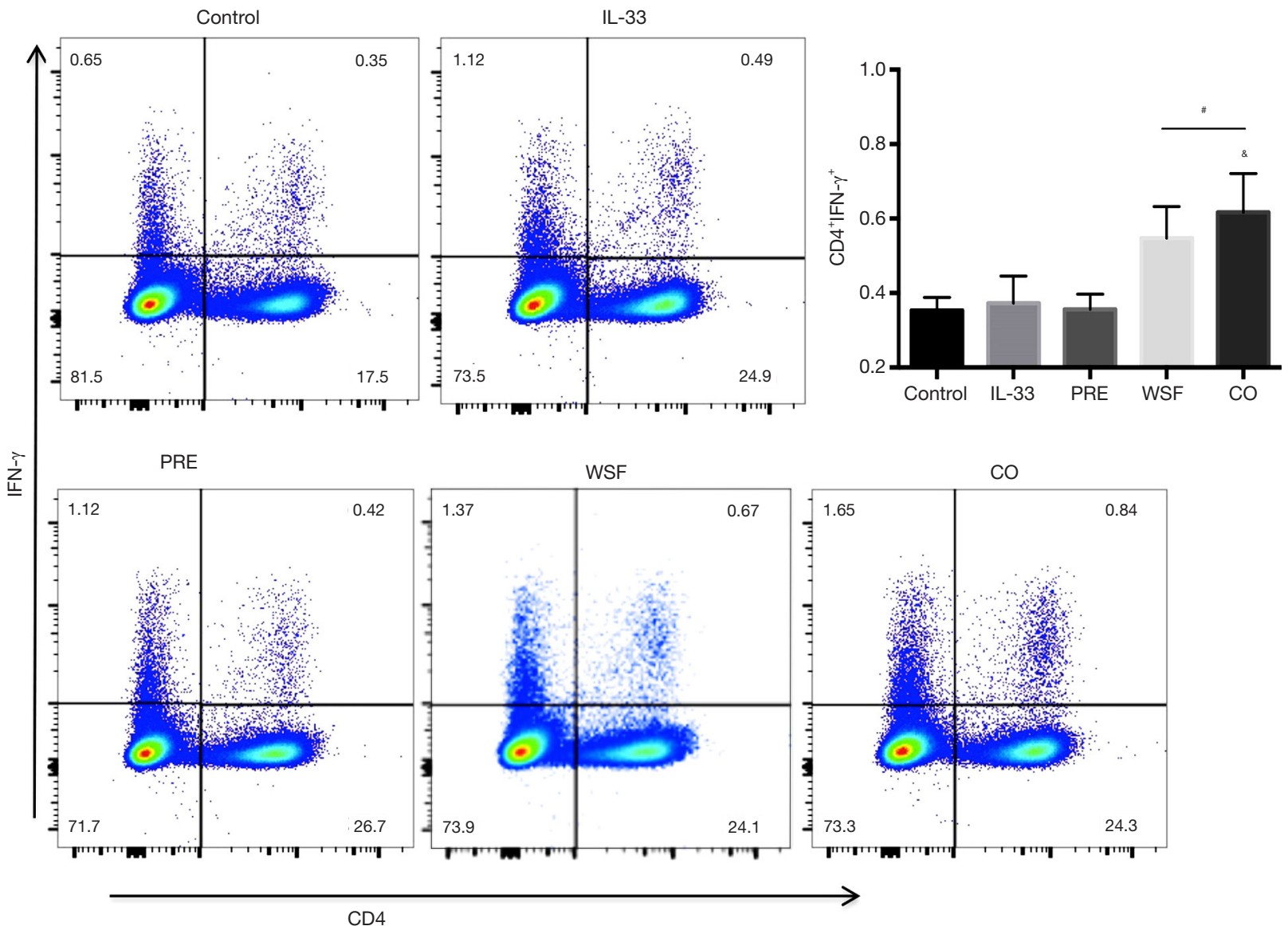

Figure 8 Effects of Wenshen decoction on IFN- $\gamma$ expression in the spleen. Data are show as means \pm SEM (n=6). ${ }^{\#} \mathrm{P}<0.05$, PRE, WSF, and CO groups vs. IL-33 group; ${ }^{\circledR} \mathrm{P}<0.05$, PRE group vs. WSF group, WSF group vs. CO group. Control, control group; IL-33, IL-33-treated group; PRE, prednisone acetate group; WSF, Wenshen decoction group; CO, prednisone and Wenshen decoction group.

model. Our results showed that Wenshen decoction effectively alleviated AHR, inhibited airway inflammation in the lung, and reduced serum $\operatorname{IgE}$ and $\mathrm{Th} 2$ type cytokines in the BALF of IL-33-induced asthma mice.

In present study, the IL-33-induced asthma model rather than the classic OVA-induced asthma model was employed based on the important role of IL-33 in the pathogenesis of asthma. IL- 33 is a member of the IL- 1 family and secreted mainly by the epithelial cells, smooth muscle cells and DCs (18). In the past decade, studies have confirmed that IL-33 is able to promote lung fibrosis and bronchial remodeling through targeting various key effector cells in the innate immune system and adaptive immune system, which further accelerates the development of asthma (36). IL-33 mainly promotes the production of type 2 cytokines. After IL-33 stimulation, Th2 cells secrete IL-4, IL-5 and IL-13, participating in the Th2 immune response (15); basophils and mast cells induce IL-4 production, inducing the $\mathrm{B}$ cells to secrete $\mathrm{IgE}$; mast cells induce IL-13 to promote the contraction of airway smooth muscle cells, causing AHR. Besides, the response to IL-33 is often related to ILC2s, which mediates the rapid production of large amounts of IL-5 and IL-13 upon IL-33 activation, playing critical roles in the pathogenesis of asthma (37).

In the IL-33 induced mouse asthma model, the antiasthmatic effect of Wenshen decoction was investigated. Our results showed the AHR of IL-33 induced asthma mice was more severe than in the control group, and different treatments could attenuate the AHR of asthmatic mice to different extents. Upon the IL-33 stimulation, a large number of inflammatory cells (mainly eosinophils) infiltrated the airway, the airway was thickened and became stenotic, mucosal folds increased, and mucus secretion increased. Different treatments could reduce the infiltrated 
inflammatory cells around the trachea, tracheal thickening was reduced, and the airway mucus production also reduced (Figure 4). After IL-33 stimulation, the contents of IL-4, IL-5 and IL-13 in the BALF significantly increased, but they were down-regulated after Wenshen decoction treatment (Figure 5).

Some studies have shown that IL-33 may induce ILC2 activation and clinical studies also reveal the IL-33 expression and ILC2 increase in the peripheral blood of asthma patients (38). Thus, we hypothesized that Wenshen decoction could reduce the immune-inflammation through regulating the ILC2. First, the influence of Wenshen decoction on the ILC2 was examined by flow cytometry. Currently, there is no specific marker for ILC2. The more surface marker molecules are lineage- (CD25, CD69, CD127, T1/T2, ICOS, KLRG1, Sca-1, IL-17BR and CD117). In the present study, LIN-ST2+KLRG1+ILC2 cells were detected. Results showed IL-33 could markedly increase the number of ILC2; in contrast, the Wenshen decoction significantly reduced the number of ILC2, though this reduction was the lowest in the Wenshen decoction group among three treatment groups. Next, the effect of Wenshen decoction on the function of ILC2 was further investigated. ROR $\alpha$ is a key transcriptional regulator in the development of ILC2. It not only mediates the immune response of ILC2 in the bone marrow and peripheral tissues, but also regulates the proliferation and biological function of ILC2. In the ROR $\alpha$ deficient mice, the expression of ILC2 is also lacking and the production of IL-4, IL-5 and IL-13 decrease, which thus impair the immune inflammatory response (39). Our study showed IL-33 could increase the mRNA expression of ROR $\alpha$, while Wenshen decoction significantly down-regulated the mRNA expression of ROR $\alpha$.

Both GATA-3 and T-bet is important transcriptional regulator, playing an important role in Th cell differentiation, which can regulate the differentiation of Th0 to Th1/Th2 in asthmatic attack. GATA-3 is mainly related to Th2 differentiation, which can promote the synthesis of Th2 cytokines, such as IL-4, IL-5 and IL-13. However, T-bet can promote Th1 differentiation, especially the synthesis of Th1 cytokines, such as IFN- $\gamma$ expression. Meanwhile, T-bet can inhibit Th2 differentiation. Studies showed decreased T-bet expression and increased GATA3 expression in asthma model of mice. Our study showed that T-bet expression decreased and GATA-3 expression increased significantly in IL-33 induced asthmatic model. After intervention with Wenshen decoction, T-bet expression was upregulated significantly, while GATA-3 expression was upregulated significantly on the contrary. This was consistent with the regulation of Wenshen decoction on inflammatory cytokines like IL-4, IL-5 and IL-13 in BALF and the regulation of IFN- $\gamma$ in this study.

Furthermore, the potential pathway related to the function of ILC2 was further investigated in IL-33induced asthma mouse model. Studies have shown that ST2 is a specific receptor for IL-33 and expressed on a variety of cells including ILC2 and DCs $(22,23)$, and IL$33 / \mathrm{ST} 2$ plays an important role in the pathogenesis of asthma. The expressions of IL-33 and ST2 are elevated in the asthmatic patients (40). The inhibition of IL-33/ ST2 has been found to improve the airway inflammation in asthmatic mice (41). Our results showed the ST2 mRNA expression significantly increased in the IL-33-treated mice, but Wenshen decoction markedly reduced the mRNA expression of ST2. It indicates that Wenshen decoction may affect the functional activation of ILC2 through the IL-33/ ST2 signaling pathway.

How the activated ILC2 affect the secreted cells? ICOS is a member of the CD28 receptor family and is mainly expressed on the DCs and B cells (26). Recent studies have shown that human and murine ILC2 expresses both ICOS and ICOS-L. ICOS/ICOSL is a main immune tolerance pathway, and has become a new target for the treatment of autoimmune diseases and allergic diseases $(42,43)$. The ICOS/ICOSL interaction is an important signal and regulator of the biological functions of ILC2. For example, it can promote the activation, function and homeostasis of ILC2, induce ILC2 to secrete cytokines and enhance AHR (27). Moreover, the interaction between ICOS and ICOSL can affect the interaction between ILC2 and other immune cells. Our results showed, in IL-33-treated mice, the mRNA expression of ICOS was up-regulated, while Wenshen decoction down-regulated the ICOS mRNA expression, suggesting that Wenshen decoction may affect the secretion of downstream cytokines by down-regulating the expression of ICOS in the activated ILC2.

IFN- $\gamma$ is a cytokine that is critical for the regulation of both innate and adaptive immune responses (44). IFN- $\gamma$ can inhibit IL-33-dependent activation of ILC2s. Interestingly, in $\mathrm{Rag}_{2}{ }^{-/-}$mice lacking $\mathrm{T}$ and $\mathrm{B}$ cells, the IFN $-\gamma$ production significantly reduces (45), but the IL-5 production dramatically increases (46). Recent findings further suggest that IFN- $\gamma$ diminishes ILC2 activity to limit excessive inflammation following asthma exacerbation after fungal infection, In available studies, 
IFN- $\gamma$ has been reported to inhibit the production of both IL- 5 and IL-13 by ILC2s $(47,48)$. Our results showed that Wenshen decoction significantly up-regulated the expression of IFN- $\gamma$, indicating that Wenshen decoction may directly suppress the activation and function of ILC2 via IFN- $\gamma$.

In conclusion, our study indicates that Wenshen decoction may inhibit the ILC2 activation through the IL33/ST2 pathway and may suppress the function of ILC2 (reductions in ILC2 and inflammatory cytokines IL-4, IL-5 and IL-13) through the ICOS/ICOSL interaction, further suppressing the airway inflammation and AHR, which improves the asthma. Besides, IFN- $\gamma$ may be a target regulated by the Wenshen decoction and may affect ILC2 in asthma. In the future, we will continue to explore the regulatory effects of Wenshen decoction on the ILC2 and IFN- $\gamma$, which will provide more evidence on the clinical use of Wenshen decoction in the treatment of asthma.

\section{Acknowledgments}

Funding: This work was supported by the National Natural Science Program of China (No. 81573937).

\section{Footnote}

Conflicts of Interest: The authors have no conflicts of interest to declare.

Ethical Statement: The authors are accountable for all aspects of the work in ensuring that questions related to the accuracy or integrity of any part of the work are appropriately investigated and resolved. The animal care and protocol were approved by the Animal Experiment Administration Committee of Shanghai Municipal Hospital of TCM (No. 2019DWLL002).

\section{References}

1. Lambrecht BN, Hammad H. The immunology of asthma. Nat Immunol 2015;16:45-56.

2. Papi A, Brightling C, Pedersen SE, et al. Asthma. Lancet 2018;391:783-800.

3. KleinJan A. Airway inflammation in asthma: key players beyond the Th2 pathway. Curr Opin Pulm Med 2016;22:46-52.

4. Lambrecht BN, Hammad H, Fahy JV. The Cytokines of Asthma. Immunity 2019;50:975-91.
5. Kim HY, Umetsu DT, Dekruyff RH. Innate lymphoid cells in asthma: Will they take your breath away? Eur J Immunol 2016;46:795-806.

6. Thiriou D, Morianos I, Xanthou G, et al. Innate immunity as the orchestrator of allergic airway inflammation and resolution in asthma. Int Immunopharmacol 2017;48:43-54.

7. Bernink JH, Germar K, Spits H. The role of ILC2 in pathology of type 2 inflammatory diseases. Curr Opin Immunol 2014;31:115-20.

8. Martinez-Gonzalez I, Steer CA, Takei F. Lung ILC2s link innate and adaptive responses in allergic inflammation. Trends Immunol 2015;36:189-95.

9. Halim TY, Hwang YY, Scanlon ST, et al. Group 2 innate lymphoid cells license dendritic cells to potentiate memory TH2 cell responses. Nat Immunol 2016;17:57-64.

10. Li BW, de Bruijn MJ, Tindemans I, et al. T cells are necessary for ILC2 activation in house dust mite-induced allergic airway inflammation in mice. Eur J Immunol 2016;46:1392-403.

11. Mortaz E, Amani S, Mumby S, et al. Role of Mast Cells and Type 2 Innate Lymphoid (ILC2) Cells in Lung Transplantation. J Immunol Res 2018;2018:2785971.

12. Rajput C, Cui T, Han M, et al. RORalpha-dependent type 2 innate lymphoid cells are required and sufficient for mucous metaplasia in immature mice. Am J Physiol Lung Cell Mol Physiol 2017;312:L983-93.

13. Han M, Rajput C, Hong JY, et al. The Innate Cytokines IL-25, IL-33, and TSLP Cooperate in the Induction of Type 2 Innate Lymphoid Cell Expansion and Mucous Metaplasia in Rhinovirus-Infected Immature Mice. J Immunol 2017;199:1308-18.

14. Cayrol C, Girard JP. Interleukin-33 (IL-33): A nuclear cytokine from the IL-1 family. Immunol Rev 2018;281:154-68.

15. Hilvering B, Xue L, Pavord ID. IL-33-dependent Th2 response after rhinovirus infection in asthma: more information needed. Am J Respir Crit Care Med 2015;191:237.

16. Lund SJ, Portillo A, Cavagnero K, et al. Leukotriene C4 Potentiates IL-33-Induced Group 2 Innate Lymphoid Cell Activation and Lung Inflammation. J Immunol 2017;199:1096-104.

17. Park SH, Kim MS, Lim HX, et al. IL-33-matured dendritic cells promote Th17 cell responses via IL-1beta and IL-6. Cytokine 2017;99:106-13.

18. Drake LY, Kita H. IL-33: biological properties, functions, and roles in airway disease. Immunol Rev 2017;278:173-84.

19. Bahrami Mahneh S, Movahedi M, Aryan Z, et al. 
Serum IL-33 Is Elevated in Children with Asthma and Is Associated with Disease Severity. Int Arch Allergy Immunol 2015;168:193-6.

20. Raeiszadeh Jahromi S, Mahesh PA, Jayaraj BS, et al. Serum levels of IL-10, IL-17F and IL-33 in patients with asthma: a case-control study. J Asthma 2014;51:1004-13.

21. Dinarello CA. Overview of the IL-1 family in innate inflammation and acquired immunity. Immunol Rev 2018;281:8-27.

22. Takatori H, Makita S, Ito T, et al. Regulatory Mechanisms of IL-33-ST2-Mediated Allergic Inflammation. Front Immunol 2018;9:2004.

23. Wang D, Bai S, Cui Y, et al. Respiratory syncytial virus prevents the subsequent development of ovalbumininduced allergic responses by inhibiting ILC2 via the IL33/ST2 pathway. Immunotherapy 2018;10:1065-76.

24. Duerr CU, Fritz JH. Isolation of Group 2 Innate Lymphoid Cells from Mouse Lungs. Methods Mol Biol 2017;1656:253-61.

25. Halim TY, Steer CA, Matha L, et al. Group 2 innate lymphoid cells are critical for the initiation of adaptive T helper 2 cell-mediated allergic lung inflammation. Immunity 2014;40:425-35.

26. Wikenheiser DJ, Stumhofer JS. ICOS Co-Stimulation: Friend or Foe? Front Immunol 2016;7:304.

27. Maazi H, Patel N, Sankaranarayanan I, et al. ICOS:ICOSligand interaction is required for type 2 innate lymphoid cell function, homeostasis, and induction of airway hyperreactivity. Immunity 2015;42:538-51.

28. Wei Y, Liu B, Sun J, et al. Regulation of Th17/Treg function contributes to the attenuation of chronic airway inflammation by icariin in ovalbumin-induced murine asthma model. Immunobiology 2015;220:789-97.

29. Li B, Duan X, Xu C, et al. Icariin attenuates glucocorticoid insensitivity mediated by repeated psychosocial stress on an ovalbumin-induced murine model of asthma. Int Immunopharmacol 2014;19:381-90.

30. Chiang CY, Lee CC, Fan CK, et al. Osthole treatment ameliorates Th2-mediated allergic asthma and exerts immunomodulatory effects on dendritic cell maturation and function. Cell Mol Immunol 2017. [Epub ahead of print].

31. Wang J, Fu Y, Wei Z, et al. Anti-asthmatic activity of osthole in an ovalbumin-induced asthma murine model. Respir Physiol Neurobiol 2017;239:64-9.

32. Bui TT, Piao CH, Song $\mathrm{CH}$, et al. Piper nigrum extract ameliorated allergic inflammation through inhibiting Th2/ Th17 responses and mast cells activation. Cell Immunol
2017;322:64-73.

33. Ji NF, Xie YC, Zhang MS, et al. Ligustrazine corrects Th1/Th2 and Treg/Th17 imbalance in a mouse asthma model. Int Immunopharmacol 2014;21:76-81.

34. Liu F, Yu J, Bai L, et al. Pingchuan formula improves asthma via restoration of the Th17/Treg balance in a mouse model. BMC Complement Altern Med 2015;15:234.

35. Reddel HK, Levy ML. The GINA asthma strategy report: what's new for primary care? NPJ Prim Care Respir Med 2015;25:15050.

36. Johansson K, McSorley HJ. Interleukin-33 in the developing lung-Roles in asthma and infection. Pediatr Allergy Immunol 2019;30:503-10.

37. Csoka B, Nemeth ZH, Duerr CU, et al. Adenosine receptors differentially regulate type 2 cytokine production by IL-33-activated bone marrow cells, ILC2s, and macrophages. Faseb J 2018;32:829-37.

38. Christianson CA, Goplen NP, Zafar I, et al. Persistence of asthma requires multiple feedback circuits involving type 2 innate lymphoid cells and IL-33. J Allergy Clin Immunol 2015;136:59-68.e14.

39. Wong SH, Walker JA, Jolin HE, et al. Transcription factor RORalpha is critical for nuocyte development. Nat Immunol 2012;13:229-36.

40. Mitchell PD, Salter BM, Oliveria JP, et al. IL-33 and Its Receptor ST2 after Inhaled Allergen Challenge in Allergic Asthmatics. Int Arch Allergy Immunol 2018;176:133-42.

41. Lee HY, Rhee CK, Kang JY, et al. Blockade of IL-33/ST2 ameliorates airway inflammation in a murine model of allergic asthma. Exp Lung Res 2014;40:66-76.

42. Lee HJ, Kim SN, Jeon MS, et al. ICOSL expression in human bone marrow-derived mesenchymal stem cells promotes induction of regulatory T cells. Sci Rep 2017;7:44486.

43. Maazi H, Akbari O. ICOS regulates ILC2s in asthma. Oncotarget 2015;6:24584-5.

44. Capobianchi MR, Uleri E, Caglioti C, et al. Type I IFN family members: similarity, differences and interaction. Cytokine Growth Factor Rev 2015;26:103-11.

45. Sun K, Metzger DW. Inhibition of pulmonary antibacterial defense by interferon-gamma during recovery from influenza infection. Nat Med 2008;14:558-64.

46. Gorski SA, Hahn YS, Braciale TJ. Group 2 innate lymphoid cell production of IL-5 is regulated by NKT cells during influenza virus infection. PLoS Pathog 2013;9:e1003615.

47. Duerr CU, McCarthy CD, Mindt BC, et al. Type I 
interferon restricts type 2 immunopathology through the regulation of group 2 innate lymphoid cells. Nat Immunol 2016;17:65-75.

48. Moro K, Kabata H, Tanabe M, et al. Interferon and IL-

Cite this article as: Huang W, Song Y, Wang L. Wenshen decoction suppresses inflammation in IL-33-induced asthma murine model via inhibiting ILC2 activation. Ann Transl Med 2019;7(20):570. doi: 10.21037/atm.2019.09.34
27 antagonize the function of group 2 innate lymphoid cells and type 2 innate immune responses. Nat Immunol 2016;17:76-86. 